OPEN ACCESS

Edited by:

Yi Su,

Banner Alzheimer's Institute,

United States

Reviewed by:

Antonina Luca,

University of Catania, Italy

Kewei Chen,

Banner Alzheimer's Institute,

United States

*Correspondence:

Gyeong-Moon Kim

kimgm@skku.edu

Specialty section:

This article was submitted to

Dementia and Neurodegenerative

Diseases,

a section of the journal

Frontiers in Neurology

Received: 13 March 2020

Accepted: 25 August 2020

Published: 29 September 2020

Citation:

Sim JE, Park M-S, Shin H-Y,

Jang $H$-S, Won H-H, Seo SW

Seo W-K, Kim BJ and Kim G-M (2020)

Correlation Between Hippocampal

Enlarged Perivascular Spaces and

Cognition in Non-dementic Elderly

Population. Front. Neurol. 11:542511.

doi: 10.3389/fneur.2020.542511

\section{Correlation Between Hippocampal Enlarged Perivascular Spaces and Cognition in Non-dementic Elderly Population}

\author{
Jae Eun Sim ${ }^{1}$, Moo-Seok Park ${ }^{2}$, Hee-Young Shin ${ }^{3}$, Hyun-Soon Jang ${ }^{4}$, Hong-Hee Won ${ }^{5}$, \\ Sang Won Seo ${ }^{6}$, Woo-Keun Seo ${ }^{6}$, Byoung Joon Kim ${ }^{6}$ and Gyeong-Moon Kim ${ }^{6 *}$ \\ ${ }^{1}$ Department of Neurology, Geumcheon Su Hospital, Seoul, South Korea, ${ }^{2}$ Department of Neurology, Seoul Medical Center, \\ Seoul, South Korea, ${ }^{3}$ Department of Health Promotion Center, Samsung Medical Center, Sungkyunkwan University School \\ of Medicine, Seoul, South Korea, ${ }^{4}$ Department of Neurology, Anseong St. Mary Hospital, Anseong, South Korea, \\ ${ }^{5}$ Department of Health Sciences and Technology, Sungkyunkwan University School of Medicine, Suwon-si, South Korea, \\ ${ }^{6}$ Department of Neurology, Samsung Medical Center, Sungkyunkwan University School of Medicine, Seoul, South Korea
}

Background and aims: The pathophysiology of hippocampal enlarged perivascular spaces (H-EPVS) and its relationship to cognitive impairment is largely unknown. This study aimed to investigate the relationship between H-EPVS and cognition in non-dementic elderly population.

Methods: A total of 109 subjects were prospectively enrolled. The eligibilities for inclusion were age from 55 to 85 years and Mini-Mental Status Examination score of $\geq 26$. The Alzheimer's Disease Assessment Scale-Cognitive Subscale (ADAS-Cog), Montreal Cognitive Assessment, transcranial Doppler (TCD), and brain magnetic resonance imaging results were evaluated. $\mathrm{H}$-EPVS was categorized in a three-degree scale: degree 0 (no), degree $1(1,2)$, and degree 2 (>2). The associations between H-EPVS and TCD parameters/cognitive test profiles were analyzed.

Results: The mean age was 65.2 years, and 52.3\% subjects were men. H-EPVS was found to be associated with age (degree 2 vs. degree 1 vs. degree 0, 69.20 \pm 6.93 vs. $65.70 \pm 5.75$ vs. $63.80 \pm 5.43 ; p=0.030$ ) and ADAS-Cog memory score (degree 2 vs. degree 1 vs. degree $0,14.88 \pm 4.27$ vs. $12.49 \pm 4.56$ vs. 11.4 \pm 4.23; $p=0.037$ ). However, the pulsatility index was not related to the degree of $\mathrm{H}$-EPVS. Multivariate analysis revealed medial temporal atrophy (MTA) scale score was independently associated with ADAS-Cog memory score (MTA scale sum $\geq 4, p=0.011$ ) but not with the degree of H-EPVS. MTA scale score showed correlation with H-EPVS $(r=0.273, p=0.004)$.

Conclusions: Aging was associated with the development of H-EPVS in non-dementic elderly population. Memory function was found to be associated with MTA but not with the degree of H-EPVS.

Keywords: hippocampal perivascular enlarged space, pulsatility index, Alzheimer's disease assessment scale-cognitive subscale (ADAS-cog), memory, medial temporal atrophy 


\section{INTRODUCTION}

The majority of patients who developed dementia had a smaller baseline hippocampal volume before their clinical diagnosis than individuals who remained free from dementia (1). While hippocampal volumes have been extensively examined in patients with cognitive impairment, signal variation within the hippocampus commonly observed on magnetic resonance imaging (MRI), described as hippocampal enlarged perivascular spaces (H-EPVS), have received less attention. Various radiological terminologies have been previously used for this feature $(2,3)$. Recently, it has been called an enlarged perivascular space (4-6). The prevalence of hippocampal enlarged perivascular spaces (H-EPVS) seems to about more than half in healthy elderly populations (2-4). However, the risk factors of H-EPVS, their radiological relevance, and their impact on cognitive performance remain under-investigated until now.

The severity of basal ganglia enlarged perivascular spaces (BG-EPVS) or white matter enlarged perivascular spaces (WMEPVS) was recently found to be associated with an increased risk of cognitive decline or incident dementia (7). The BGEPVS is more strongly linked to markers of small vessel disease in patients with stroke and hypertensive vasculopathy in patients with cognitive impairment $(1,8-10)$. The WM-EPVS was associated with incident dementia in healthy subjects (11). Centrum semi-ovale EPVS severity is strongly associated with clinically diagnosed Alzheimer's disease (AD)-related cognitive impairment (12). However, the association between the degree of H-EPVS and cognitive impairment is controversial. Previous studies have investigated the effect of H-EPVS on cognition, but the results were inconsistent. Considering H-EPVS, the results are not associated with cognitive ability or development of dementia $(2,3,13)$. Higher H-EPVS counts were linked to better cognitive performance in memory (5). There are fewer studies on the relationship of H-EPVS and cognitive impairment. H-EPVS was associated with global cognitive function measured using the Mini-Mental State Examination (MMSE) (14) and worse verbal reasoning function (4). Further investigation of H-EPVS and cognition in larger cohorts without cerebrovascular and amyloid burden (e.g., healthy elderly cohorts) will help clarify the correlation of H-EPVS and cognition.

It has been suggested that EPVS is a pathologic hallmark of compromised fluid and toxin clearance from the brain and a potential biomarker of neurovascular and neurodegenerative diseases (15). Parenchymal amyloid $\beta$ could reach the vessel walls through perivascular spaces, eventually aggregating within them and causing retrograde perivascular enlargement due to interstitial space fluid blockage $(10,16)$. The vascular pathology causes a decrease in vessel wall compliance, increaseing cerebrovascular pulsatility. The change in cerebrovascular pulsatility affects glymphatic homeostasis and brain clearance $(10,17)$. Impaired vascular circulation from any cause leads to cognitive impairment $(18,19)$. The middle cerebral arterypulsatility index (MCA-PI) is independently associated with cognitive impairment in middle-aged asymptomatic subjects (20). Patients with AD with higher posterior atrophy scores have worse performance on tasks of visuospatial and executive function (21).

We hypothesized that the degree of H-EPVS is related to increased arterial PI and cognitive impairment. This study aimed to investigate risk factors of H-EPVS and the relationship between $\mathrm{H}$-EPVS and PI or cognition particularly memory domain in non-dementic elderly population.

\section{MATERIALS AND METHODS \\ Subjects}

Subjects were prospectively recruited from the outpatients who underwent brain MRI and transcranial Doppler (TCD) in the Samsung Medical Center health promotion center between October 2015 and August 2017. Then subjects were clinically evaluated and underwent cognition test, The Alzheimer's Disease Assessment Scale-Cognitive Subscale (ADAS-Cog), MMSE, and Montreal Cognitive Assessment (MoCA). The eligibilities for inclusion were age from 55 to 85 years and MMSE score of $\geq 26$. Individuals with evidence of dementia, structural brain alternation including mass, stroke, and psychiatric problems were excluded. The complete study protocol was approved by the Ethical Committee of the Sungkyunkwan University. All subjects provided written informed consent. The following clinical variables were recorded for each subject: age, sex, education, smoking, alcohol consumption, and presence of hypertension, diabetes, and hypercholesterolemia. Education was defined as years of formal education. Smoking habit was categorized as current smoker or ex-smoker/never smoker. Hypertension was defined as previous diagnosis of hypertension (>140/90 $\mathrm{mmHg}$ ) or use of antihypertensive treatment for control of blood pressure. Diabetes was defined as previous diagnosis of diabetes or current use of antidiabetic drugs. Hypercholesterolemia was defined as previous diagnosis of hypercholesterolemia or current use of antihyperlipidemic medications. We examined the blood for urea and electrolyte level, calcium level, liver function, glucose level, hemoglobin level, white cell count, platelet count, and cholesterol level.

\section{Image Acquisition}

Each patient underwent 3-Tesla MRI (Achieva; Philips Medical System, Best, the Netherlands) including diffusion-weighted imaging, conventional T1- and T2-weighted imaging, T2 fluid attenuated inversion recovery imaging, gradient echo imaging, 3D time-of-flight magnetic resonance angiography (MRA), and contrast enhanced MRA. Three-Tesla MRI (Achieva; Philips Medical System, Best, the Netherlands) was used to acquire 3D T1 turbo field echo MRI data with the following imaging parameters: sagittal slice thickness, $1.0 \mathrm{~mm}$; over contiguous slices with $50 \%$ overlap; no gap; repetition time, $9.9 \mathrm{~ms}$; echo time, $4.6 \mathrm{~ms}$; flip angle, $8^{\circ}$; and matrix size of $240 \times 240$ pixels reconstructed to $480 \times 480$ over a field of view of $240 \mathrm{~mm}$.

\section{TCD Protocol}

TCD (Pioneer TC 8080; Nicolet Vascular, Madison, WI, USA) was used to monitor both MCAs with insonation depths of $40-60 \mathrm{~mm}$ and both posterior cerebral arteries (PCAs) with 

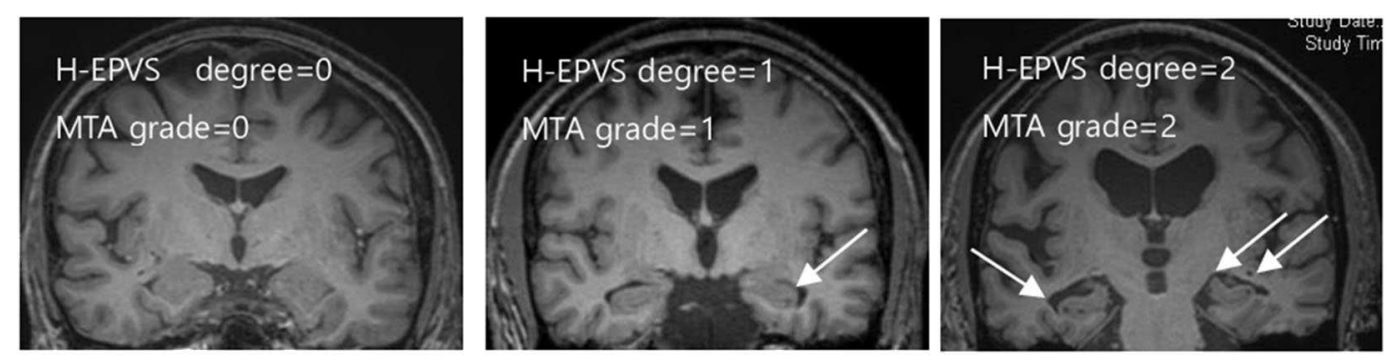

FIGURE 1 | Examples of the degree of H-EPVS and MTA scale score. The three MRI images include each degree of H-EPVS and MTA scale score. White arrows point examples of H-EPVS. H-EPVS, hippocampal enlarged perivascular spaces; MTA, medial temporal atrophy.

insonation depths of $60-70 \mathrm{~mm}$. We followed the TCD criteria based on consensus criteria of the fourth meeting of the European Society of Neurosonology and Cerebral Hemodynamics. The TCD recording quality was continuously observed by one investigator. Data were automatically saved on the computer hard disk for review, and all analyses were performed blinded to the individual patient details.

\section{Rating of H-EPVS}

H-EPVS were defined as CSF-like signal-intensity change (hypointense on T1 and hyperintense on T2) that were round, curvilinear, or crescent, $<3 \mathrm{~mm}$ in their maximum diameter (22), with smooth delineated contours, and typically located in the lateral portion of the hippocampus (2). H-EPVS were identified on T1 coronal images. For lesions fulfilling the abovementioned criteria but having maximum diameter $\geq 3 \mathrm{~mm}$, only those with a typical vascular shape and following the orientation of perforating arteries were diagnosed (11).

H-EPVS were individually and separately scored on the right and left side of the hippocampus by two experienced neurologists who were blinded to all clinical data. Two independent raters blinded to each other's readings rated H-EPVS manually. In case of disagreement, a consensus meeting was held. The sum of bilateral sides was used later for statistical analysis. We categorized in a three-degree scale (3) according to the sum of $\mathrm{H}$ EPVS in the left and right hippocampus: degree 0 (no), degree 1 $(1,2)$, and degree $2(>2)$. Intra- and inter-observer agreement was assessed using a weighted Cohen $\kappa$ statistics. $\kappa$-value was 0.759 . Figure 1 demonstrates H-EPVS example of each severity degree of both hippocampi.

\section{Hippocampal Atrophy}

Semiquantitative analysis of hippocampal atrophy by medial temporal atrophy (MTA) scale score was administered. The image analysis included a visual rating of MTA on the T1weighted images. T1-weighted images were viewed in the coronal plane, and MTA scale scores for the left and right hemispheres were provided. MTA was rated on a 5-point scale (0 point, absent; 1 point, minimal; 2 points, mild; 3 points, moderate; and 4 points, severe) based on the height of the hippocampal formation and width of the choroid fissure and temporal horn. The sum of the MTA scale scores from both hemispheres were calculated for the statistical analysis. Quantitative analysis of hippocampal volume was estimated using Inbrain ${ }^{\circledR}$ software (https://www.inbrain.co.kr/) $(23,24)$. Based on FreeSurfer 6.0, the hippocampal volume values of Inbrain ${ }^{\circledR}$ software are the same. The sum of both hippocampal volumes were calculated for the statistical analysis.

\section{Cognitive Assessment}

Cognitive function was assessed using the ADAS-Cog test that included several domains. All tests were conducted by the same tester who was blinded to the results of brain imaging and biochemistry. ADAS-Cog is the most widely used general cognitive measure in clinical trials of $\mathrm{AD}$. We divided into subgroups by cognitive domain $(25,26)$. For the memory domain including an orientation part, we checked word recall of 12 items, word delayed recall, and word recognition. For the language function, we checked naming, remembering test instructions, spoken language ability, word finding difficulty, and comprehension. For praxia, we checked commands, constructional praxia, and ideational praxia. A higher ADAS-Cog score reflected lower performance. We also performed MMSE and MoCA to assess global cognition. Finally, the geriatric depression scale was administered to assess the presence of depression.

\section{Statistical Analysis}

Initially, we divided the H-EPVS count and categorized it into three degrees. To study the risk factors associated with $\mathrm{H}$ EPVS, we performed appropriate univariate tests to compare clinical and radiologic data between subjects with high and low degrees of H-EPVS. Continuous values are adjusted to mean \pm standard deviation. The ANOVA and Kruskal Wallis test were applied for continuous variables, while the $\chi^{2}$-test and Fisher's exact test were applied to categorical variables. After we found that ADAS-Cog memory score was related to the degree of H-EPVS, particularly degree 2, we divided subjects into two groups by ADAS-Cog memory to study the risk factors associated with memory cognitive function. The $t$-test and Wilcoxon rank sum test were applied to continuous variables, while the $\chi^{2}$-test and Fisher's exact test were applied to categorical variables. The association of memory cognitive function and degree of H-EPVS, MTA scale sum 
score and hippocampal total volume was determined using linear regression to obtain effect estimates and standard errors. Then, adjustments for covariates were constructed initially for age, sex, education, high-density lipoprotein (HDL), and hypertension, which are variables showing differences in the univariate analysis $(p<0.1)$ and subsequently for degree of $\mathrm{H}$ EPVS, MTA scale sum score and hippocampal total volume. We used the $\mathrm{R}$ software ( $\mathrm{R}$ version 3.6.5, The $\mathrm{R}$ Foundation for Statistical Computing). Two-tailed $p<0.05$ were considered statistically significant.

\section{RESULTS}

\section{Baseline Characteristics}

In a 2-year period, 109 subjects were enrolled. The overall demographics, clinical characteristics, blood chemistry results, and H-EPVS count are presented in Table 1. The mean age of subjects was 65.2 years $(\mathrm{SD}=5.94)$, and $52.3 \%(n=57)$ subjects were men. Sixty-two (56.9 \%) individuals had at least $1 \mathrm{H}$-EPVS with a maximum number of 5 . Degree 1 was the most frequent H-EPVS degree, accounting for $45.9 \%$ of cases. Figure 1 shows the representative MRI of each degree of H-EPVS.

\section{Risk Factor of H-EPVS}

The demographics, laboratory results, cognitive function, and brain imaging findings of the degree of H-EPVS are presented in Table 2. The degree of H-EPVS was associated with age (degree 2 vs. degree 1 vs. degree $0,69.20 \pm 6.93$ vs. 65.70 \pm 5.75 vs. $63.80 \pm 5.43, p=0.030$ ). In the ordinary logistic regression analysis for association between $\mathrm{H}$-EPVS and age, $P$-value was 0.006 . Odds ratio was 1.095 and $95 \%$ confidence interval was 1.028-1.169. The degree of H-EPVS was related to ADAS-Cog memory score (degree 2 vs. degree 1 vs. degree $0: 14.88 \pm 4.27$ vs. $12.49 \pm 4.56$ vs. $11.40 \pm 4.23$, $p$-value $0.037)$. MTA scale sum score was increased with increasing degree of H-EPVS (degree 2 vs. degree 1 vs. degree 0 , $2.50 \pm 1.45$ vs. $1.34 \pm 1.44$ vs. $1.06 \pm 1.39 ; p=0.005)$. H-EPVS and MTA scale score, hippocampal total volume showed the correlation (Figure 2, correlation efficient, 0.273; $p=0.004$; correlation efficient, $0.199 ; p=0.038$ ). In contrast, the degree of H-EPVS had no association with sex, smoking, or alcohol drinking status, hypercholesterolemia, diabetes, coronary heart disease, and depression. $P$-value of current smoking was 0.094 .

\section{Degree of H-EPVS and PI and Mean Flow Velocity of the Middle Cerebral Artery and Posterior Cerebral Artery}

The degree of H-EPVS was not found to be related to PI and mean flow velocity of the MCA and PCA (data not shown). Although there was a trend toward increased PI values with $\mathrm{H}$ EPVS degree, the $p$-values of the right PCA PI were 0.058 (P1) and 0.084 (P2).

\section{Risk Factor of Memory Cognitive Function}

To study the risk factor associated with memory cognitive function, we divided subjects into two groups by ADAS-Cog
TABLE 1 | Baseline characteristics of the study subjects.

\begin{tabular}{|c|c|}
\hline Characteristics $(n=109)$ & \\
\hline Age (year) (range) & $65.2 \pm 5.94(55-85)$ \\
\hline Male sex, $n(\%)$ & $57(52.3)$ \\
\hline Education (year) & $12.4 \pm 3.75$ \\
\hline Hypertension, $n$ (\%) & $46(42.2)$ \\
\hline Diabetes, $n(\%)$ & $17(15.6)$ \\
\hline Hyperlipidemia, n (\%) & $50(45.9)$ \\
\hline Coronary artery disease, $n(\%)$ & $12(11)$ \\
\hline Depression, $n$ (\%) & $5(4.6)$ \\
\hline Current smoking, $n(\%)$ & $11(10.1)$ \\
\hline Current alcohol consumption, $n$ (\%) & $58(53.2)$ \\
\hline $\mathrm{SBP}(\mathrm{mmHg})$ & $125 \pm 16.0$ \\
\hline DBP (mmHg) & $72.8 \pm 8.91$ \\
\hline FBS (mg/dL) & $102 \pm 17.6$ \\
\hline T-Chol (mg/dL) & $186 \pm 38.4$ \\
\hline LDL (mg/dL) & $122 \pm 36.7$ \\
\hline $\mathrm{HDL}(\mathrm{mg} / \mathrm{dL})$ & $55.1 \pm 13.0$ \\
\hline TG (mg/dL) & $124 \pm 61.8$ \\
\hline \multicolumn{2}{|l|}{ Number of H-EPVS } \\
\hline 0 & 47 \\
\hline $1-2$ & 50 \\
\hline$>2$ & 12 \\
\hline GDS & $2.73 \pm 2.93$ \\
\hline MMSE & $28.5 \pm 1.29$ \\
\hline MoCA & $25.9 \pm 2.55$ \\
\hline MoCA memory & $2.64 \pm 1.68$ \\
\hline ADAS-Cog & $15.2 \pm 5.46$ \\
\hline ADAS-Cog-language & $0.339 \pm 0.748$ \\
\hline ADAS-Cog-memory & $12.2 \pm 4.45$ \\
\hline ADAS-Cog-praxia & $2.52 \pm 1.60$ \\
\hline
\end{tabular}

Key: SBP, systolic blood pressure; DBP, diastolic blood pressure; FBS, fasting blood sugar; T-chol, total cholesterol; LDL, low-density lipoprotein; HDL TG, triglyceride; $\mathrm{H}$ EPVS, hippocampal enlarged perivascular spaces; GDS, geriatric depression scale; MMSE, Mini-Mental State Examination; MoCA, Montreal Cognitive Assessment; ADASCog, The Alzheimer's Disease Assessment Scale-Cognitive Subscale.

memory score (Table 3 , score $\geq 9$ vs. score $<9$ ). In univariate analysis, ADAS-Cog memory score was associated with age (score $\geq 9$ vs. score $<9,66.00 \pm 6.10$ vs. $63.00 \pm 4.85 ; p=0.025$ ) and education (score $\geq 9$ vs. score $<9,12.0 \pm 3.82$ vs. $13.7 \pm 3.82$; $p=0.034$ ). ADAS-Cog memory score was associated with $\mathrm{H}$ EPVS count, MTA scale sum score and hippocampal total volume (score $\geq 9$ vs. score $<9,1.11 \pm 1.22$ vs. $0.593 \pm 0.797 ; p=0.041$; score $\geq 9$ vs. score $<9,1.51 \pm 0.690$ vs. $0.852 \pm 0.949 ; p=0.042$; score $\geq 9$ vs. score $<9,7,878 \pm 867$ vs. $8,255 \pm 775$; $p=0.047$ ). ADAS-Cog memory score was also associated with degree 2 of $\mathrm{H}$-EPVS and MTA scale sum score $\geq 4(p=0.035 ; p=0.034)$.

\section{Independent Contributions on Memory Cognitive Function}

Considering multivariate models to determine predictors of ADAS-Cog memory score, MTA scale score was found to be independently associated with ADAS-Cog memory score 
TABLE 2 | Demographic, laboratory results, cognitive function, and brain imaging findings according to the degree of H-EPVS.

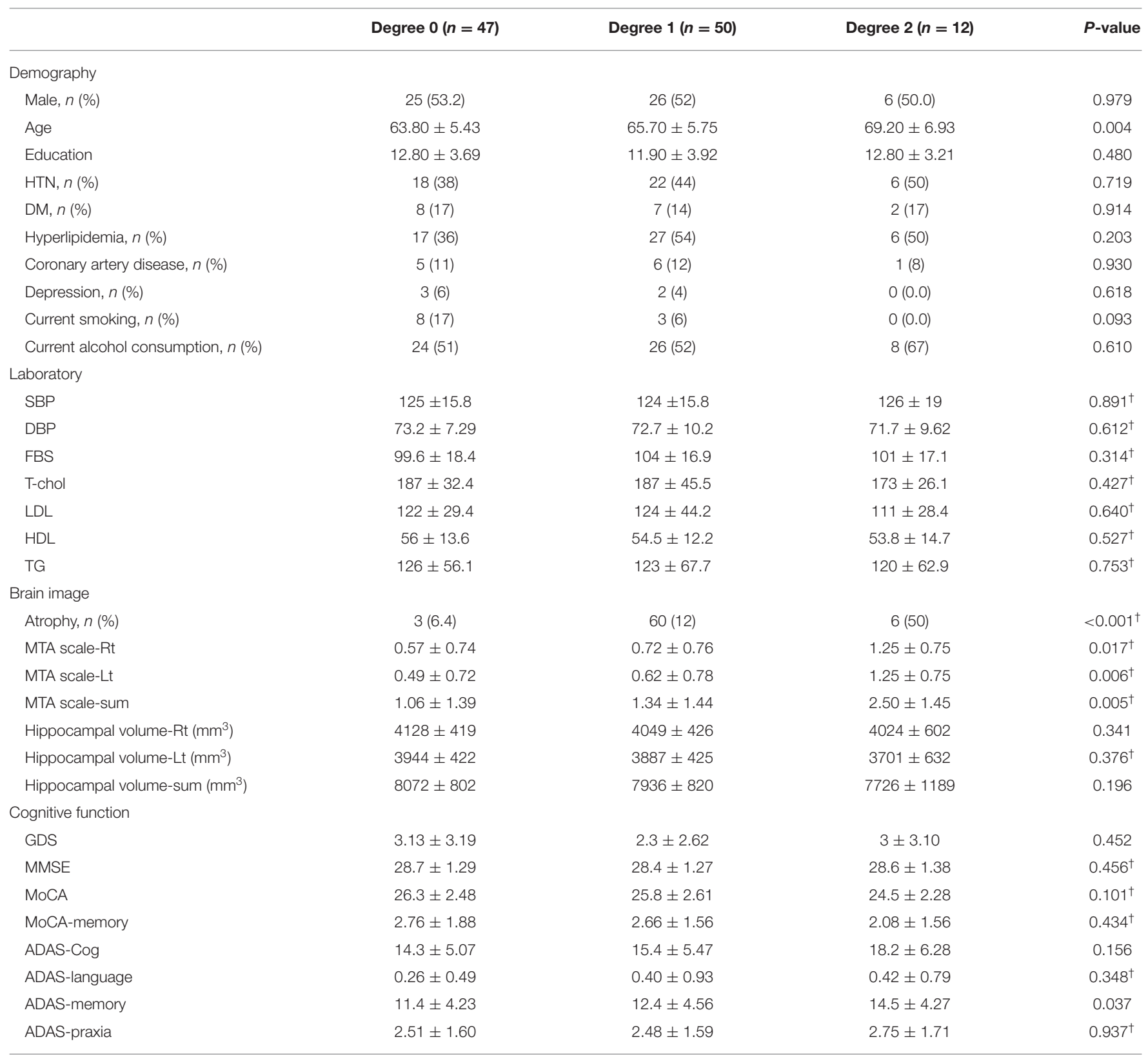

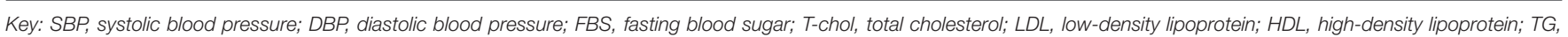

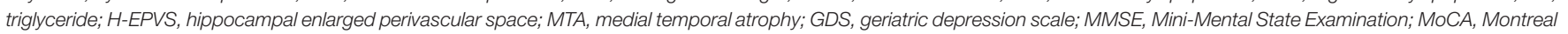
Cognitive Assessment; ADAS-Cog, The Alzheimer's Disease Assessment Scale-Cognitive Subscale ${ }^{\dagger}$ Kruskal Wallis test.

[Table 4, MTA scale sum score $\geq 4$, $p$-value $0.011, \beta$ (95\% CI) $3.467(0.802-6.133)]$. However, after adjustments for age, sex, education, HDL, and hypertension, the degree of H-EPVS and hippocampal total volume were not found related to ADASCog memory score (Table 4, degree 2, $p=0.113 ; p=0.090$ ). With adjustment of multivariable factors, education duration was found to be independently associated with memory cognitive function (Table 4, $p<0.05$ ). We showed the correlation of memory cognitive function and H-EPVS count, MTA scale sum score and hippocampal total volume (Figure 3).

\section{DISCUSSION}

In this prospective study of 109 subjects, we found the age plays a key role in the development of H-EPVS in non-dementic elderly population. Besides, memory cognitive function was found to be independently associated with MTA but not with the degree of H-EPVS.

Enlarged perivascular spaces commonly referred to as the Virchow-Robin space, which was originally described by Durand Fardel in 1843, detailed by Rudolf Virchow in 1851 (27), and later 


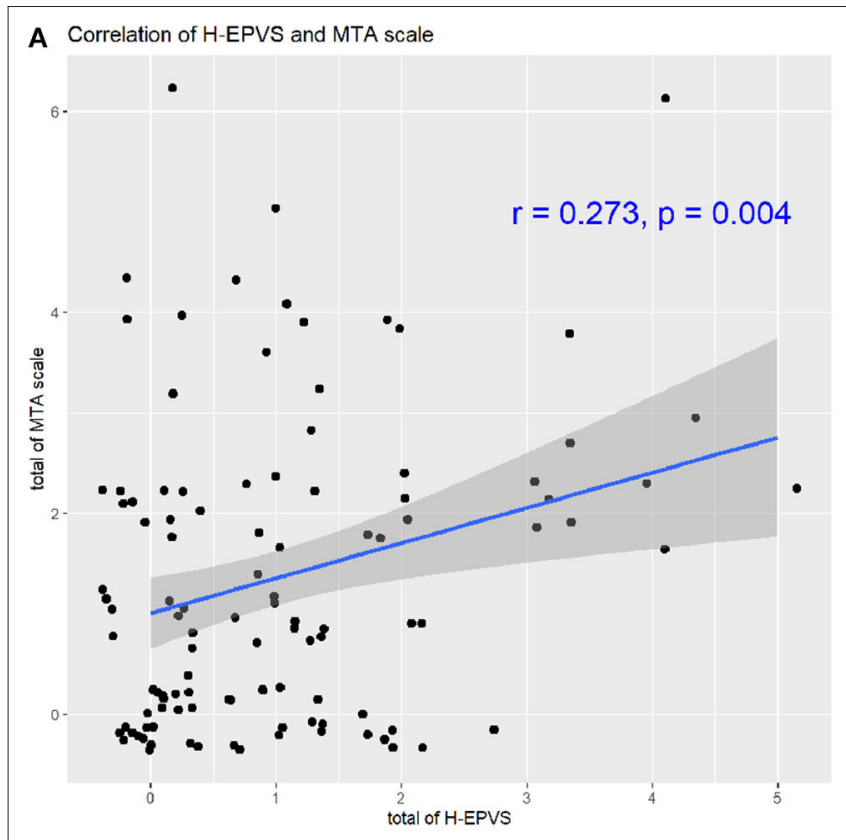

B Correlation of H-EPVS and Hippocampal total volume

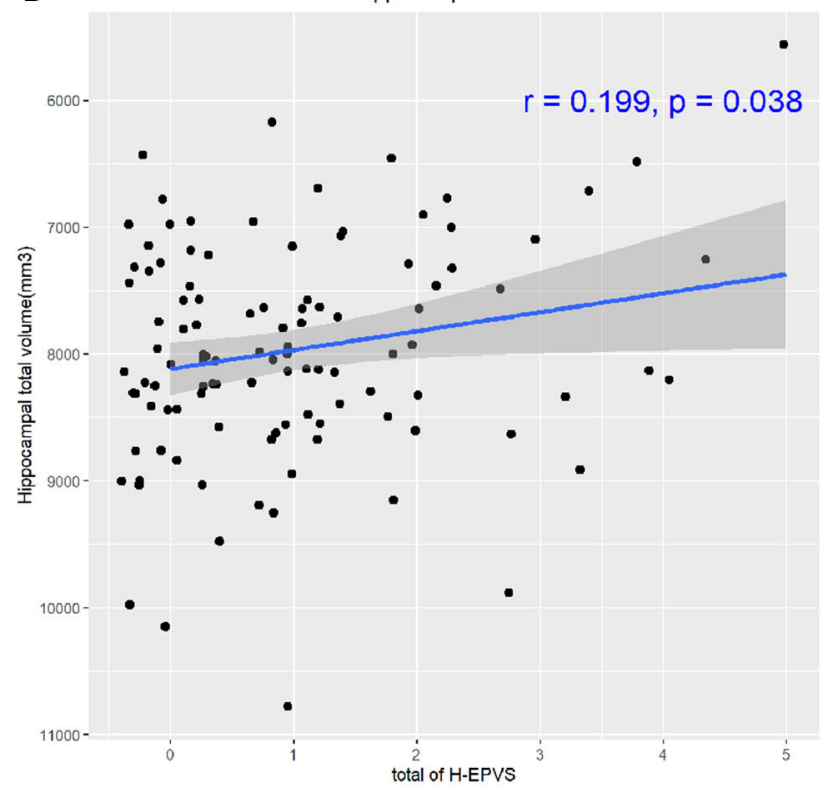

FIGURE 2 | Correlation of H-EPVS and hippocampal atrophy. The line in each graph was obtained from the univariate regression model. $\mathrm{R}$ means correlation coefficients. Correlation of H-EPVS and MTA scale (A). Correlation of H-EPVS and hippocampal total volume (B). H-EPVS, hippocampal enlarged perivascular spaces; MTA, medial temporal atrophy. confirmed by Charles-Philippe Robin as donut-shaped channels surrounding the brain's blood vessels (28). It is believed to be caused by extensions of the subarachnoid space surrounding the penetrating arteries (29). The compromised fluid and toxin clearance from the brain is a causal role of EPVS. We investigated
TABLE 3 | Demographic, laboratory results, and imaging features of the subjects according to ADAS-Cog memory score.

\begin{tabular}{|c|c|c|c|}
\hline & $\begin{array}{c}\text { ADAS-Cog } \\
\text { memory <9 } \\
(n=27)\end{array}$ & $\begin{array}{c}\text { ADAS-Cog } \\
\text { memory } \geq 9 \\
(n=82)\end{array}$ & $P$-value \\
\hline \multicolumn{4}{|l|}{ Demography } \\
\hline Male, $n(\%)$ & $12(44)$ & $45(55)$ & 0.472 \\
\hline Age & $63 \pm 4.85$ & $66 \pm 6.10$ & 0.025 \\
\hline Education & $13.7 \pm 3.24$ & $12.0 \pm 3.82$ & $0.034^{\ddagger}$ \\
\hline $\mathrm{HTN}, n(\%)$ & $7(26)$ & $39(48)$ & 0.080 \\
\hline $\mathrm{DM}, n(\%)$ & $2(7)$ & $15(18)$ & 0.295 \\
\hline Hyperlipidemia, $n$ (\%) & $13(48)$ & $37(45)$ & 0.959 \\
\hline Coronary artery disease, $n(\%)$ & $3(11)$ & $9(11)$ & $>0.999$ \\
\hline Depression, $n(\%)$ & $1(4)$ & $4(5)$ & $>0.999$ \\
\hline Current smoking, $n(\%)$ & $5(19)$ & $6(7)$ & 0.191 \\
\hline Current alcohol consumption, $n(\%)$ & $13(48)$ & $45(55)$ & 0.700 \\
\hline \multicolumn{4}{|l|}{ Laboratory } \\
\hline SBP & $123 \pm 12.3$ & $125 \pm 17.1$ & 0.621 \\
\hline DBP & $71.9 \pm 8.33$ & $73.1 \pm 9.12$ & 0.535 \\
\hline FBS & $102 \pm 14.3$ & $102 \pm 18.6$ & $0.918^{\ddagger}$ \\
\hline T-chol & $189 \pm 40.8$ & $184 \pm 37.8$ & 0.596 \\
\hline LDL & $120 \pm 40.7$ & $122 \pm 35.7$ & $0.797^{\ddagger}$ \\
\hline $\mathrm{HDL}$ & $61.7 \pm 14.1$ & $52.9 \pm 11.9$ & 0.002 \\
\hline $\mathrm{TG}$ & $123 \pm 41.2$ & $124 \pm 67.5$ & $0.934^{\ddagger}$ \\
\hline \multicolumn{4}{|l|}{ H-EPVS } \\
\hline \multirow[b]{5}{*}{ Degree $\geq 2$} & & & 0.042 \\
\hline & $16(59)$ & $31(38)$ & \\
\hline & $11(41)$ & $39(47)$ & \\
\hline & $0(0)$ & $12(15)$ & \\
\hline & $0(0)$ & $12(15)$ & 0.035 \\
\hline Total count of H-EPVS & $0.593 \pm 0.797$ & $1.11 \pm 1.22$ & 0.041 \\
\hline \multicolumn{4}{|l|}{ Hippocampal atrophy } \\
\hline Atrophy, $n(\%)$ & $0(0)$ & $15(18)$ & 0.038 \\
\hline MTA scale-Rt & $0.444 \pm 0.506$ & $0.805 \pm 0.823$ & 0.034 \\
\hline MTA scale-Lt & $0.407 \pm 0.572$ & $0.707 \pm 0.824$ & 0.082 \\
\hline MTA scale sum & $0.852 \pm 0.949$ & $1.51 \pm 0.690$ & 0.042 \\
\hline MTA scale sum $\geqq 4$ & $0(0)$ & $13(16)$ & 0.034 \\
\hline Hippocampal volume-Rt $\left(\mathrm{mm}^{3}\right)$ & $4,236 \pm 415$ & $4,029 \pm 442$ & 0.035 \\
\hline Hippocampal volume-Lt $\left(\mathrm{mm}^{3}\right)$ & $4,019 \pm 398$ & $3,849 \pm 462$ & $0.054^{\ddagger}$ \\
\hline Hippocampal volume-sum $\left(\mathrm{mm}^{3}\right)$ & $8255 \pm 775$ & $7878 \pm 867$ & 0.047 \\
\hline
\end{tabular}

Key: SBP, systolic blood pressure; DBP, diastolic blood pressure; FBS, fasting blood sugar; T-chol, total cholesterol; LDL, low-density lipoprotein; HDL, high-density lipoprotein; TG, triglyceride; H-EPVS, hippocampal enlarged perivascular spaces; ADASCog, The Alzheimer's Disease Assessment Scale-Cognitive Subscale; MTA, medial temporal atrophy ${ }^{\ddagger}$ Wilcoxon rank sum test.

whether the degree of H-EPVS is related to increased arterial PI and cognitive impairment.

A similar age effect was previously detected in previous studies $(3,4,30,31)$. Moreover, EPVS become increasingly common with aging $(32,33)$. Hypertension and poor treatment compliance of hypertension were independently related to the large number of H-EPVS $(3,4,30)$. However, our study found no relationship between hypertension and degree of H-EPVS. H-EPVS was 
TABLE 4 | The impact of H-EPVS, MTA and hippocampal volume on ADAS-Cog memory.

\begin{tabular}{|c|c|c|}
\hline & \multicolumn{2}{|l|}{ ADAS-Cog memory } \\
\hline & $\beta(95 \% \mathrm{Cl})$ & $p$-value \\
\hline \multicolumn{3}{|c|}{ H-EPVS and ADAS-Cog memory } \\
\hline \multicolumn{3}{|l|}{ Model 1} \\
\hline Degree of $\mathrm{H}$-EPVS $\geq 2$ & $2.142(-0.515$ to 4.799$)$ & 0.113 \\
\hline Age & $0.123(-0.025$ to 0.272$)$ & 0.101 \\
\hline Sex (male) & $1.244(-0.536$ to 3.024$)$ & 0.169 \\
\hline Education & $-0.314(-0.547$ to -0.082$)$ & 0.009 \\
\hline HDL & $-0.027(-0.090$ to 0.037$)$ & 0.409 \\
\hline HTN & $0.292(-1.447$ to 2.031$)$ & 0.740 \\
\hline \multicolumn{3}{|c|}{ MTA scale and ADAS-Cog memory } \\
\hline \multicolumn{3}{|l|}{ Model 2} \\
\hline MTA scale sum $\geq 4$ & 3.467 (0.802 to 6.133$)$ & 0.011 \\
\hline Age & $0.086(-0.065$ to 0.236$)$ & 0.261 \\
\hline Sex (male) & $0.816(-0.947$ to 2.579$)$ & 0.361 \\
\hline Education & $-0.287(-0.516$ to -0.059$)$ & 0.014 \\
\hline HDL & $-0.022(-0.085$ to 0.041$)$ & 0.490 \\
\hline $\mathrm{HTN}$ & $0.242(-1.464$ to 1.947$)$ & 0.780 \\
\hline \multicolumn{3}{|c|}{ Hippocampal volume and ADAS-Cog memory } \\
\hline \multicolumn{3}{|l|}{ Model 3} \\
\hline Hippocampal volume & $-0.001(-0.002$ to 0.0001$)$ & 0.090 \\
\hline Age & $0.108(-0.045$ to 0.260$)$ & 0.163 \\
\hline Sex (male) & $1.722(-0.169$ to 3.613$)$ & 0.074 \\
\hline Education & $-0.297(-0.529$ to -0.065$)$ & 0.013 \\
\hline HDL & $-0.027(-0.091$ to 0.036$)$ & 0.396 \\
\hline HTN & $-0.033(-1.806$ to 1.793$)$ & 0.970 \\
\hline
\end{tabular}

Multivariable linear regression analyses with covariates (Age, Sex, Education, HDL, HTN) were built to assess the association between ADAS-Cog-memory and degree of H-EPVS and hippocampal atrophy. In each model, ADAS-Cog-memory score was the outcome and degree of H-EPVS, MTA sum score and hippocampal volume.

Key: H-EPVS, hippocampal enlarged perivascular spaces; HDL, high-density lipoprotein; MTA, medial temporal atrophy score; ADAS-Cog, The Alzheimer's Disease Assessment Scale-Cognitive Subscale.

observed in $56.9 \%$ of individuals. This result is similar to those of previous studies (2-4).

The memory cognitive function and association of the degree of H-EPVS are controversial. In previous studies, the degree of $\mathrm{H}$-EPVS does not appear to be associated with the development of dementia or cognitive ability $(3,11,13)$. A meta-analysis of five population-based studies reported that higher H-EPVS counts were linked to better cognitive performance in memory (5). We divided subjects into two groups by ADAS-Cog memory score (score $\geq 9$ vs. score $<9$ ) to determine the risk factors of memory cognitive function. In multivariate models to determine predictors of ADAS-Cog memory score, MTA scale score were found to be independently associated with ADAS-Cog memory score (MTA scale sum score $\geq 4, p=0.011$ ). However, the degree of H-EPVS was not found to be related to ADAS-Cog memory score (degree 2, $p=0.113$ ). This result means that the risk factor of memory cognitive function is related to MTA but not the degree of H-EPVS.
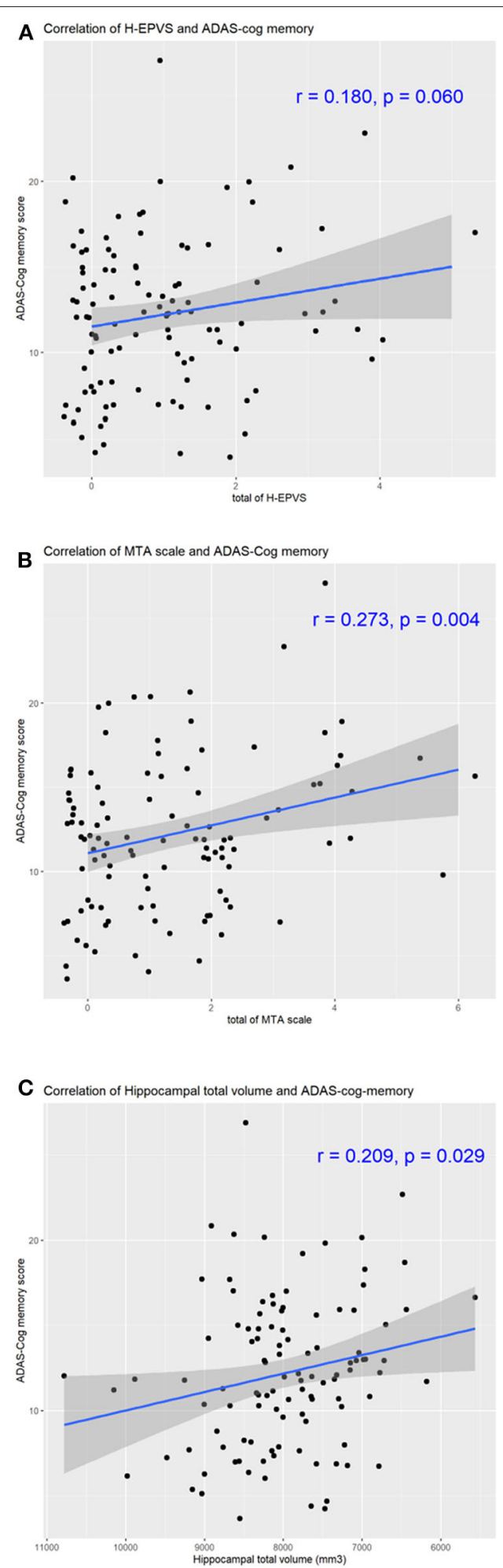

FIGURE 3 | Correlation of H-EPVS, hippocampal atrophy and cognitive function. The line in each graph was obtained from the univariate regression model. $\mathrm{R}$ means correlation coefficients. Correlation of H-EPVS and ADAS-Cog memory (A). Correlation of MTA scale and ADAS-Cog memory (B). Correlation of hippocampal total volume and ADAS-Cog memory (C). H-EPVS, hippocampal enlarged perivascular spaces; MTA, medial temporal atrophy; ADAS-Cog, The Alzheimer's Disease Assessment Scale-Cognitive Subscale. 
In our study, we found that the degree of H-EPVS was associated with MTA scale score. We showed the correlation of H-EPVS and MTA scale score, hippocampal total volume (Figure 2, correlation efficient, 0.273; $p=0.004$; correlation efficient, $0.199 ; p=0.038$ ). Therefore, we believe that $\mathrm{H}$ EPVS may be a secondary phenomenon of MTA. However, it is not completely clear whether H-EPVS follows the development of clinically overt MTA. Future studies with larger-scale longitudinal designs are warranted to examine whether MTA contributes to H-EPVS. The relationship between EPVS and brain atrophy is controversial. Brain atrophy is an independent risk factor for severe EPVS in the basal ganglia $(34,35)$. In contrast to our study, other studies reported the association of the degree of H-EPVS and higher volume of the hippocampus. Another study reported that H-EPVS was not influenced by hippocampal atrophy (2). Because HEPVS was associated with age in our study, there may be a possibility that MTA is associated with H-EPVS even in non-dementic subjects.

With adjustment of multivariable factors, education duration was found to be independently associated with memory cognitive function $(p<0.05)$. In previous studies, more years of education moderate genetic risk and memory function associated with amyloid $\beta$ by increasing cognitive function capacity $(36,37)$.

We failed to find any significant association between the degree of H-EPVS and cerebrovascular pulsatility. Shi et al. reported that high PI was only associated with basal ganglia but not centrum semi-ovale PVS (31). They explained that the arterioles in the two locations showed differences in associations of PVS. PI showed difficulty in assessing the resistance of small vessel and reflecting microvascular pathology. Moreover, our study participants have lesser vascular risk factors. Our results showed that the $p$-values of right PCA PI were 0.058 and 0.084 , which are more related to the degree of H-EPVS than MCA values. It may be because the origin of hippocampal arteries is the PCA or PCA branches, but further study with large population is needed (38).

The strength of our study is that we performed detailed cognitive tests compared with previously published papers. They used simple cognitive tests, such as MMSE, Isaacs set tests, or trail making tests $(3,5,13,30)$, which are crude measures of cognitive function. However, the ADAS$\mathrm{Cog}$ is more accurate, both in differentiating individuals with normal cognition from those with impaired cognition and in assessing the extent of cognitive impairment in individuals. With the imaging study in healthy non-dementic subjects, we could study the correlation of H-EPVS and cognitive ability with lesser confounding factors, such as white matter hyperintensity, lacunar infarcts, and brain atrophy.

However, our study has several limitations. First, the use of a semiquantitative evaluation for rating the degree of H-EPVS can limit our ability to detect small effects and differences. Extremely small ischemic cavities in the cerebral tissue might have been misclassified as EPVS. Future advances in automatic/semiautomatic computational segmentation algorithms for EPVS quantification may improve crossvalidation between studies (39). For hippocampal atrophy, we used the semiquantitative and quantitative analyses. But in multivariate models to determine predictors of ADASCog memory score, results were inconsistent $(p=0.011$ vs. $p=0.090)$. It is because our participants were nondementic and smaller group. Hippocampal volume is useful only in distinguishing late onset $\mathrm{AD}$ and amnestic-early onset $\mathrm{AD}$ from healthy population (40). So, it is difficult to find differences of memory score by hippocampal volume in this group. Third, it is well-known that the hippocampus plays an important role in declarative and episodic memory. ADAS-Cog memory subscales principally evaluate verbal memory. Therefore, it would be more appropriate to use a more complete memory scale with other tests. Fourth, we did not consider the relationship between H-EPVS and other radiologic markers of cognitive impairment, such as total brain volume and cortical thickness. Fifth, our study has a cross-sectional design, which restricts our interpretation of data with respect to cause and consequence. Finally, PI and MFV were not available in $18 \%$ of subjects due to poor temporal window. We excluded their data from the analysis, which may result in selection bias in that the excluded individuals may be older, may be less educated, and may carry more vascular risk factors.

\section{CONCLUSION}

The present findings suggest that age plays a key role in the development of H-EPVS in the non-dementic elderly population. The degree of H-EPVS was found be associated with MTA scale score. However, TCD parameters were not related to the degree of H-EPVS. With adjustment of multivariable factors, memory cognitive function was found to be independently associated with MTA, but not with the degree of H-EPVS.

\section{DATA AVAILABILITY STATEMENT}

The original contributions presented in the study are included in the article/supplementary material, further inquiries can be directed to the corresponding author/s.

\section{ETHICS STATEMENT}

The studies involving human participants were reviewed and approved by the Ethical Committee of the Sungkyunkwan University. The patients/participants provided their written informed consent to participate in this study.

\section{AUTHOR CONTRIBUTIONS}

All authors listed have made a substantial, direct and intellectual contribution to the work, and approved it for publication. 


\section{REFERENCES}

1. den Heijer T, van der Lijn F, Koudstaal PJ, Hofman A, van der Lugt A, Krestin GP, et al. A 10-year follow-up of hippocampal volume on magnetic resonance imaging in early dementia and cognitive decline. Brain. (2010) 133(Pt 4):1163-72. doi: 10.1093/brain/awq048

2. Li Y, Li J, Segal S, Wegiel J, De Santi S, Zhan J, et al. Hippocampal cerebrospinal fluid spaces on MR imaging: relationship to aging and alzheimer disease. AJNR Am J Neuroradiol. (2006) 27:912-8.

3. Yao M, Zhu YC, Soumare A, Dufouil C, Mazoyer B, Tzourio C, et al. Hippocampal perivascular spaces are related to aging and blood pressure but not to cognition. Neurobiol Aging. (2014) 35:2118-25. doi: 10.1016/j.neurobiolaging.2014.03.021

4. Jimenez-Balado J, Riba-Llena I, Garde E, Valor M, Gutierrez B, Pujadas F, et al. Prevalence of hippocampal enlarged perivascular spaces in a sample of patients with hypertension and their relation with vascular risk factors and cognitive function. J Neurol Neurosurg Psychiatry. (2018) 89:651-6. doi: 10.1136/jnnp-2017-316724

5. Hilal S, Tan CS, Adams HHH, Habes M, Mok V, Venketasubramanian $\mathrm{N}$, et al. Enlarged perivascular spaces and cognition: a metaanalysis of 5 population-based studies. Neurology. (2018) 91:e832-42. doi: 10.1212/WNL.0000000000006079

6. Jokinen H, Koikkalainen J, Laakso HM, Melkas S, Nieminen T, Brander A, et al. Global burden of small vessel disease-related brain changes on MRI predicts cognitive and functional decline. Stroke. (2019) 51:170-8. doi: 10.1161/STROKEAHA.119.026170

7. Leong RL, Lo JC, Sim SK, Zheng H, Tandi J, Zhou J, et al. Longitudinal brain structure and cognitive changes over 8 years in an East Asian cohort. Neuroimage. (2016) 147:852-60. doi: 10.1016/j.neuroimage.2016. 10.016

8. Doubal FN, MacLullich AM, Ferguson KJ, Dennis MS, Wardlaw JM. Enlarged perivascular spaces on MRI are a feature of cerebral small vessel disease. Stroke. (2010) 41:450-4. doi: 10.1161/STROKEAHA.109.564914

9. Rouhl RP, van Oostenbrugge RJ, Knottnerus IL, Staals JE, Lodder J. VirchowRobin spaces relate to cerebral small vessel disease severity. J Neurol. (2008) 255:692-6. doi: 10.1007/s00415-008-0777-y

10. Martinez-Ramirez S, Pontes-Neto OM, Dumas AP, Auriel E, Halpin A, Quimby $M$, et al. Topography of dilated perivascular spaces in subjects from a memory clinic cohort. Neurology. (2013) 80:1551-6. doi: 10.1212/WNL.0b013e31828f1876

11. Zhu YC, Dufouil C, Soumaré A, Mazoyer B, Chabriat H, Tzourio C. High degree of dilated Virchow-Robin spaces on MRI is associated with increased risk of dementia. J Alzheimers Dis. (2010) 22:663-72. doi: 10.3233/JAD-2010-100378

12. Banerjee G, Kim HJ, Fox Z, Jager HR, Wilson D, Charidimou A, et al. MRI-visible perivascular space location is associated with Alzheimer's disease independently of amyloid burden. Brain. (2017) 140:1107-16. doi: 10.1093/brain/awx003

13. Maclullich AM, Wardlaw JM, Ferguson KJ, Starr JM, Seckl JR, Deary IJ. Enlarged perivascular spaces are associated with cognitive function in healthy elderly men. J Neurol Neurosurg Psychiatry. (2004) 75:1519-23. doi: 10.1136/jnnp.2003.030858

14. Chen W, Song X, Zhang Y, Alzheimer's Disease Neuroimaging I. Assessment of the Virchow-Robin spaces in Alzheimer disease, mild cognitive impairment, and normal aging, using high-field MR imaging. AJNR Am J Neuroradiol. (2011) 32:1490-5. doi: 10.3174/ajnr. A2541

15. Ramirez J, Berezuk C, McNeely AA, Gao F, McLaurin J, Black SE. Imaging the perivascular space as a potential biomarker of neurovascular and neurodegenerative diseases. Cell Mol Neurobiol. (2016) 36:289-99. doi: 10.1007/s10571-016-0343-6

16. Weller RO, Hawkes CA, Kalaria RN, Werring DJ, Carare RO. White matter changes in dementia: role of impaired drainage of interstitial fluid. Brain Pathol. (2015) 25:63-78. doi: 10.1111/bpa.12218

17. Mestre H, Kostrikov S, Mehta RI, Nedergaard M. Perivascular spaces, glymphatic dysfunction, and small vessel disease. Clin Sci. (2017) 131:225774. doi: $10.1042 / C S 20160381$
18. Ruitenberg A, den Heijer T, Bakker SL, van Swieten JC, Koudstaal PJ, Hofman A, et al. Cerebral hypoperfusion and clinical onset of dementia: the rotterdam study. Ann Neurol. (2005) 57:789-94. doi: 10.1002/ana. 20493

19. Poels MM, Ikram MA, Vernooij MW, Krestin GP, Hofman A, Niessen WJ, et al. Total cerebral blood flow in relation to cognitive function: the Rotterdam scan study. J Cereb Blood Flow Metab. (2008) 28:1652-5. doi: $10.1038 / \mathrm{jcbfm} .2008 .62$

20. Lopez-Oloriz J, Lopez-Cancio E, Arenillas JF, Hernandez M, Dorado L, Dacosta-Aguayo R, et al. Diffusion tensor imaging, intracranial vascular resistance and cognition in middle-aged asymptomatic subjects. Cerebrovasc Dis. (2014) 38:24-30. doi: 10.1159/0003 63620

21. Smits LL, Tijms BM, Benedictus MR, Koedam EL, Koene T, Reuling IE, et al. Regional atrophy is associated with impairment in distinct cognitive domains in Alzheimer's disease. Alzheimers Dement. (2014) 10(Suppl. 5):S299-305. doi: 10.1016/j.jalz.2013.06.007

22. Wardlaw JM, Smith EE, Biessels GJ, Cordonnier C, Fazekas F, Frayne R, et al. Neuroimaging standards for research into small vessel disease and its contribution to ageing and neurodegeneration. Lancet Neurol. (2013) 12:822-38. doi: 10.1016/S1474-4422(13) 70124-8

23. Zhu J, Jin $\mathrm{Y}$, Wang $\mathrm{K}$, Zhou $\mathrm{Y}$, Feng $\mathrm{Y}, \mathrm{Yu}$ M, et al. Frequencydependent changes in the regional amplitude and synchronization of resting-state functional MRI in stroke. PLoS ONE. (2015) 10:e0123850. doi: 10.1371/journal.pone.0123850

24. Jung NY, Han JC, Ong YT, Cheung CY, Chen CP, Wong TY, et al. Retinal microvasculature changes in amyloid-negative subcortical vascular cognitive impairment compared to amyloid-positive Alzheimer's disease. J Neurol Sci. (2019) 396:94-101. doi: 10.1016/j.jns.2018. 10.025

25. Verma N, Beretvas SN, Pascual B, Masdeu JC, Markey MK, Alzheimer's Disease Neuroimaging I. New scoring methodology improves the sensitivity of the Alzheimer's Disease Assessment Scale-Cognitive subscale (ADAS-Cog) in clinical trials. Alzheimers Res Ther. (2015) 7:64. doi: 10.1186/s13195-015-0151-0

26. Rosen WG, Mohs RC, Davis KL. A new rating scale for Alzheimer's disease. Am J Psychiatry. (1984) 141:1356-64. doi: 10.1176/ajp.141.11.1356

27. Virchow R. Ueber die Erweiterung kleinerer Gefaesse. Archiv Pathol Anat Physiol Klin Med. (1851) 3:427-62. doi: 10.1007/BF01960918

28. Robin C. Recherches sur quelques particularites de la structure des capil-laires de l'encephale. J Physiol Homme Anim. (1859) 2:537-48.

29. Braffman BH, Zimmerman RA, Trojanowski JQ, Gonatas NK, Hickey WF, Schlaepfer WW. Brain MR-pathologic correlation with gross and histopathology. 1. Lacunar infarction and Virchow-Robin spaces. Am J Roentgenol. (1988) 151:551-8. doi: 10.2214/ajr.151.3.551

30. Zhang C, Chen Q, Wang Y, Zhao X, Wang C, Liu L, et al. Risk factors of dilated Virchow-Robin spaces are different in various brain regions. PLoS ONE. (2014) 9:e105505. doi: 10.1371/journal.pone.0105505

31. Shi Y, Thrippleton MJ, Blair GW, Dickie DA, Marshall I, Hamilton I, et al. Small vessel disease is associated with altered cerebrovascular pulsatility but not resting cerebral blood flow. J Cereb Blood Flow Metab. (2020) 40:85-99. doi: 10.1177/0271678X18803956

32. Heier LA, Bauer CJ, Schwartz L, Zimmerman RD, Morgello S, Deck MD. Large virchow-robin spaces: MR-clinical correlation. AJNR Am J Neuroradiol. (1989) 10:929-36.

33. Zhu YC, Tzourio C, Soumare A, Mazoyer B, Dufouil C, Chabriat H. Severity of dilated Virchow-Robin spaces is associated with age, blood pressure, and MRI markers of small vessel disease: a population-based study. Stroke. (2010) 41:2483-90. doi: 10.1161/STROKEAHA.110.591586

34. Zhang X, Ding L, Yang L, Qin W, Yuan J, Li S, et al. Brain atrophy correlates with severe enlarged perivascular spaces in basal ganglia among lacunar stroke patients. PLOS ONE. (2016) 11:e0149593. doi: 10.1371/journal.pone. 0149593

35. Potter GM, Doubal FN, Jackson CA, Chappell FM, Sudlow CL, Dennis MS, et al. Enlarged perivascular spaces and cerebral small vessel disease. Int $J$ Stroke. (2015) 10:376-81. doi: 10.1111/ijs.12054 
36. Vonk JMJ, Arce Rentería M, Medina VM, Pericak-Vance MA, Byrd GS, Haines J, et al. Education moderates the relation between apoe epsilon 4 and memory in nondemented non-hispanic black older adults. J Alzheimers Dis. (2019) 72:495-506. doi: 10.3233/JAD190415

37. Joannette M BC, Dupont PS, Lavallée MM, Nikelski J, Vallet GT, Chertkow H, et al. Education as a moderator of the relationship between episodic memory and amyloid load in normal aging. J Gerontol A Biol Sci Med Sci. (2019) glz235. doi: 10.1093/gerona/glz235

38. Spallazzi M, Dobisch L, Becke A, Berron D, Stucht D, Oeltze-Jafra S, et al. Hippocampal vascularization patterns: a high-resolution 7 Tesla timeof-flight magnetic resonance angiography study. Neuroimage Clin. (2019) 21:101609. doi: 10.1016/j.nicl.2018.11.019

39. Hernandez Mdel C, Piper RJ, Wang X, Deary IJ, Wardlaw JM. The automatic computational assessment of enlarged perivascular spaces on brain magnetic resonance images: a systematic review. J Magn Reson Imaging. (2013) 38:77485. doi: $10.1002 /$ jmri.24047
40. Falgas N, Sanchez-Valle R, Bargallo N, Balasa M, Fernandez-Villullas G, Bosch $\mathrm{B}$, et al. Hippocampal atrophy has limited usefulness as a diagnostic biomarker on the early onset Alzheimer's disease patients: a comparison between visual and quantitative assessment. Neuroimage Clin. (2019) 23:101927. doi: 10.1016/j.nicl.2019.101927

Conflict of Interest: The authors declare that the research was conducted in the absence of any commercial or financial relationships that could be construed as a potential conflict of interest.

Copyright (c) 2020 Sim, Park, Shin, Jang, Won, Seo, Seo, Kim and Kim. This is an open-access article distributed under the terms of the Creative Commons Attribution License (CC BY). The use, distribution or reproduction in other forums is permitted, provided the original author(s) and the copyright owner(s) are credited and that the original publication in this journal is cited, in accordance with accepted academic practice. No use, distribution or reproduction is permitted which does not comply with these terms. 